\title{
EFFICIENT USE OF HYBRID ADAPTIVE NEURO-FUZZY INFERENCE SYSTEM COMBINED WITH NONLINEAR DIMENSION REDUCTION METHOD IN PRODUCTION PROCESSES
}

\author{
Saad Bashir Alvi, Robert Martin and Johannes Gottschling \\ Mathematics for Engineer, Duisburg-Essen University, Duisburg
}

\begin{abstract}
This research study proposes a novel method for automatic fault prediction from foundry data introducing the so-called Meta Prediction Function (MPF). Kernel Principal Component Analysis (KPCA) is used for dimension reduction. Different algorithms are used for building the MPF such as Multiple Linear Regression (MLR), Adaptive Neuro Fuzzy Inference System (ANFIS), Support Vector Machine (SVM) and Neural Network (NN). We used classical machine learning methods such as ANFIS, SVM and NN for comparison with our proposed $M P F$. Our empirical results show that the MPF consistently outperform the classical methods.
\end{abstract}

\section{KEYWORDS}

Fuzzy Inference System, ANFIS, Neural Network, Support Vector Machine, KPCA

\section{INTRODUCTION}

Automatic fault prediction is an important topic of research in metal industry [1]. Since the beginning of the first industrial revolution, industries are striving to produce fault-free products in least possible amount of time. Customer expectations are ever-increasing in term of quality and availability of products [2-4]. A fault in a product during the production process can be due to a single cause or a combination of causes and industries are still using trial and error methods to minimize them[5][6]. Faults in production can be reduced by analysis of the production process data [7][8]. Data capturing of the production processes for analysis started manually but due to human-errors, the quality of the data captured was compromised. Now, with the automation of data capturing using sensors, the noisy data is getting reduced. With the advancement of the technology and automation, more and more data is available for analysis to optimise the production processes [9].

In the current globalized industry era with emphasis on automated smart industries, the analysis of measured data plays an important role to improve the quality of the products [10]. Large

Dhinaharan Nagamalai et al. (Eds) : ITCCMA, CSITY, AIFZ, NWCOM, SIGPRO - 2017

pp. 29- 43, 2017. @ CS \& IT-CSCP 2017

DOI : $10.5121 /$ csit.2017.70703 
number of parameters have an influence on the quality of a product. Deviations of process parameters can have negative effect on the production performance. Measuring and evaluating all the appropriate process parameters with a suitable method ensure a consistently high quality and productivity in the automated production environment. The application of Machine Learning (ML) methods in these processes is motivated mainly by two objectives: the prediction of quality properties from the measured data and the identification of key process indicators, i.e. the process parameters which have the strongest influence on the outcome. These objectives help to improve the quality of the products and to understand the implicit relations among the parameters in the production processes, which in turn result in reducing faulty products during production.

In this work, we will concentrate on the first objective, namely the prediction of quality properties from the measured data by suggesting a novel methodology for automatic fault prediction. The learnt methodology will help to reach target predictions, which will ensure stability of the production process regulation and repeatability of the process conditions resulting in quality products with minimal scrap. The potential of the proposed methodology is demonstrated by using three actual production datasets.

In our approach, we use three stage process. In first stage, the input data is split into mutually exclusive sets of training, validation and test data. In the second stage, Support Vector Machine (SVM) [11][12] and Neural Network (NN) [13][14] are trained using the original data whereas Adaptive Neuro Fuzzy Inference System (ANFIS) [15] is trained on the data transformed by Kernel Principal Component Analysis (KPCA) [16][17]. In the last stage, we utilize a novel fusion method to combine these different prediction functions and obtain a "Meta Prediction Function" (MPF). Results during these stages are also collected and performance is measured. Figure 1 shows major data processing units of proposed framework.

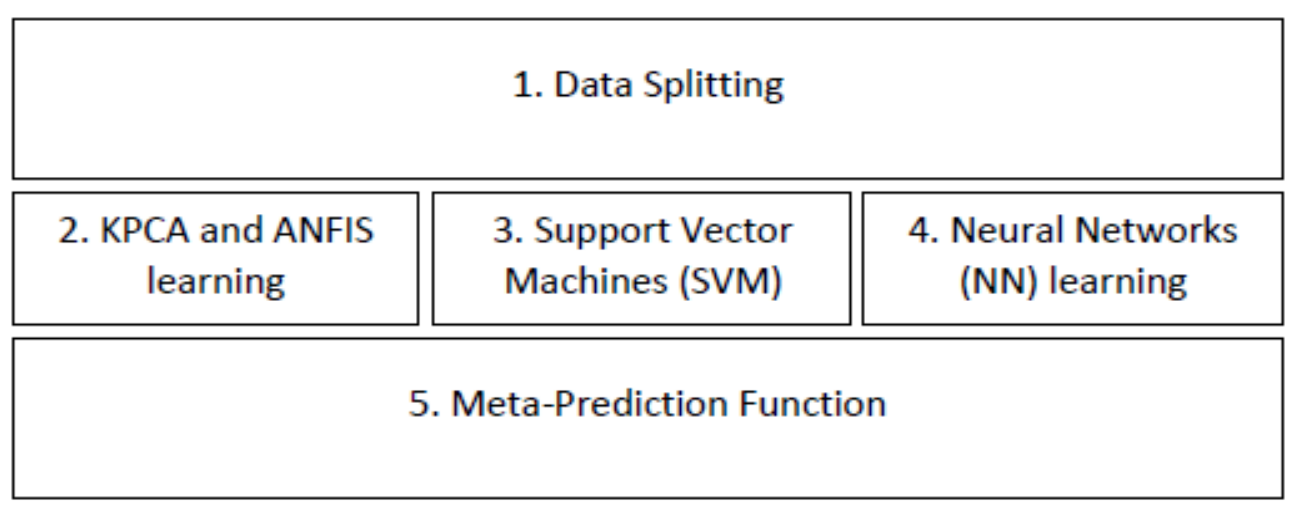

Figure 1 - Major data processing blocks

The performance of the MPF is compared with the performance of the classical machine learning methods such as Neural Networks, SVM and ANFIS.

\section{RELATED WORK}

Lehaet al proposed a novel integrated method into a production plan realized in a physics-based realistic simulator. Supervised machine learning techniques namely Model Trees and Neural Networks were integrated. The online learning and on the fly control code modification were allowed by integrating the learning capability into the control process. Averaging was used for 
measuring the produced optimization times through learning which outperform times of a production process. [18]

Ashouret al proposed a method for automated identification for machined surfaces in manufacturing.Image processing and computer vision technologies were used for automated identification for reduction in inspection time and avoidance of human error due to inconsistency and fatigue. SVM classifier with differentkernels were investigated for the categorization of machined surfaces into six machining processes. The gray level histogram was used as discriminating feature. Experimental results suggested that the SVM with the linear kernel outperformed for a dataset consisting of seventy-two workpiece images. [19]

Yuan et al proposed a novel method for improving the machining quality of thin walled flexible workpieces. Machining platform was established for thin-walled flexible workpieces. Sparse Bayesian learning based method was used to predict the future deformation. The dual mode predictive controller was developed to reduce the machining vibrations and quality of the workpiece surface was improved. Experiments were performed for thin-walled flexible workpieces and effectiveness of proposed method were demonstrated through machining experiments. [20]

Rostamiet al proposed an efficient Equipment Condition Diagnosis (ECD) model for Fault Detection and Classification (FDC). Different machine learning techniques such as SVM, KMeans clustering and Self-Organizing Map (SOM) were used to develop ECD model. Principal Component Analysis (PCA) was used to project the abnormal observations into normal models. Experiments were conducted with industry data. The proposed Model showed effectiveness for classifying the fault fingerprints to give evident guidelines in explaining the detected faults. [21]

Costa et al used ANFIS and CART (Classification and Regression Tree) for classifying the defects that occur during the production process for packing of glass. The database project was named as "Newglass". Different operating variables of the furnace and the percentage defects in the end products of the factory model were used in experiments. Finally, ANFIS, CART and glass packing manufacturing results were compared. [22]

\section{METHODOLOGY}

\subsection{Support Vector Machine}

Support Vector Machine (SVM) is a supervised learning method[11][12] which computes a set of hyperplanes in a higher dimensional feature space based on the given data for classification and regression problems.

For each dataset, optimal settings for the SVM method were chosen with experimentation. Input training and validation data was normalized with z-score normalization. Following kernel functions were considered for learning:-

the Polynomial kernel,

$$
k(X, Y)=\left(X^{T} Y+r\right)^{p},
$$


where $p$ is the power of the polynomial and $r$ is a shifting parameter;

the Gaussian kernel,

$$
k(X, Y)=e^{\frac{-\|X-Y\|^{2}}{2 \sigma^{2}}},
$$

where sigma $(\sigma)$ is an adjustable parameter;

Sigmoid kernel as shown in equation 3 below,

$$
k(X, Y)=\tanh \left(\gamma X^{T} Y+r\right)
$$

where rho $(\gamma)$ is the scaling parameter of the input data and $r$ is the shifting parameter controlling threshold of mapping.

The performance of the selected optimal settings was calculated on the test data and is shown in the experiments section. The predictions from SVM were used as input in the MPFdescribed in section 3.5.

\subsection{Neural Networks}

Neural Networks (NN) [13][14] are a non-linear supervised learning method based on a network of so-called neurons which are interconnected by weighted links. NN learns by adjusting the weights to optimal values based on the given training and validation data.

In this work, a NN is trained using one of the most popular backpropagation learning algorithm with multilayer perceptron topology. The used feedforward NN consisted of three layers: an input layer, one hidden layer and an output layer. The number of neurons in the input layer is equal to the total number of independent variables and the number of neurons in the output layer is equal to the number of dependent variables, whereas the hidden layer contains eight neurons for all considered datasets. The input layer receives the input from the independent variables and forwards it to all the neurons in the hidden layer. The neurons in the hidden layer apply their activation function to the weighted sum of their inputs and compute an output. The output layer then computes the predicted value for the dependent variable(s).

The back-propagation learning algorithm used here to train the multilayer network consists of two passes. In the forward pass, with randomly selected weights and the input given by the training data, the algorithm produces an output for the dependent variable(s). An error is then calculated based on the difference between predicted and actual output. In the backward pass, this error is propagated backwards through the network from the output layer to the input layer and weights in the network are modified using the delta rule. The formula used to calculate the change in weights of hidden and output layer neurons are shown in equation 4 and in equation 5 respectively:-

$$
\begin{aligned}
& \Delta w_{i j}(p)=\beta \cdot \Delta w_{i j}(p-1)+\alpha \cdot x_{i}(p) \cdot \delta_{j}(p) \\
& \Delta w_{j k}(p)=\beta \cdot \Delta w_{j k}(p-1)+\alpha \cdot y_{j}(p) \cdot \delta_{k}(p)
\end{aligned}
$$

where the indices $i, j, k$ refer to input, hidden and output layers,

$\alpha$ is the learning rate,

$\beta$ is the Momentum with a value between 0 and 1 , 
$x_{i}(p), y_{j}(p)$ are the output of neurons $i$ in the input layer and $j$ in the hidden layer at iteration $p$,

$\delta_{j}(p), \delta_{k}(p)$ are the error gradients at the neurons $j$ in hidden layer and $k$ in the output layer at iteration $p$.

During training, we used 1000 epochs. Weights of the NN were optimised using settings of learning rate 0.1 and momentum 0.2. The Unipolar Sigmoid function was used as the activation function.

During the learning phase, training and validation data is normalised. Then $\mathrm{NN}$ is trained and the parameter settings which produce best results are preserved and the performance of these settings is calculated on the test data.

The predictions from NN are also used as input in the MPF in section 3.5.

\subsection{Kernel Principal Component Analysis}

KernelPrincipal Component Analysis (KPCA) is a nonlinear dimension reduction method, introduced by Sholkoptet al.[16][17], which maps data from the input space to a lower dimensional feature space while retaining maximum possible variance in the data.

For each dataset, KPCA was applied using the Polynomial kernel and Gaussian kernel,which are defined in equations(1) and (2) respectively. For the Polynomial kernel, settings used were $p=$ $1,2,3 ; r=0$. For Gaussian kernel, the settings used were $\sigma=0.5,1.0,1.5$.

The results are shown for the kernel settings which provided the most accurate prediction results.

\subsection{Adaptive Neuro-Fuzzy Inference System}

Adaptive Neuro-Fuzzy Inference System (ANFIS) [15] is a hybrid neuro-fuzzy model which consists of 5 layers. In the first layer, for the inputs, degree of membership of the chosen membership function is computed. In the second layer, firing strength of the rules is calculated using t-norm (product for AND and maximum for OR) operators. In the third layer, computed firing strengths are normalized. Forth layer is the adaptation layer in which rule consequent parameters are computed. Fifth layer is the summation layer which computes sum of all the computed consequents. In learning phase of ANFIS, membership function parameters and rules consequent parameters are optimised.

In our approach, we use the ANFIS, which combines the advantages of fuzzy expert systems with those of classical NN, in combination with KPCA. It has already been successfully employed for data prediction in a variety of fields. KPCA was used to transform the data because for some of the selected parameter settings like input partition method: 'grid partitioning', ANFIS was not immediately applicable to the number of variables as large as 10. In addition, even in the case of small number of variables, it was observed that ANFIS produced comparatively better results for same parameter settings on the transformed data from KPCA in comparison to the original data as input.

Before the training in ANFIS start, a Fuzzy Inference System (FIS) is initialized with information about the number of input variables for the selected dataset, the selected number and type of membership functions and the number of rules. The rules for the FIS were initialized based on the Grid Partitioning (GP) method or Subtractive Clustering (SC) method. In case of GP method, 
input membership function type as Gaussian was selected, output membership function type as Linear was selected and in different independent runs for best parameter settings, number of membership functions for input parameters were selected as 2, 3 or 4. For SC method, influence radius was tested with inputs $0.1,0.2,0.3,0.4$ and 0.5 for different runs. It was trained using $\mathrm{NN}$ backpropagation algorithm and least square methods.

In case of KPCA and ANFIS, the training and validation data was collectively normalised and the corresponding symmetric kernel matrix was computed. The Eigen-values and Eigen-vectors were computed for the computed kernel matrix. Then principal components of the training and validation data were computed using the computed kernel matrix and Eigen-vectors. These principal components along with the corresponding dependent variable values were used to train ANFIS and parameter settings which produced the best result on the validation data were saved. Finally, the performance was calculated on the principal components of the test data. These predictions were also saved for later use in the MPF.

\subsection{Meta Prediction Function}

Our Meta Prediction Function(MPF) is inspired by the principle of ensemble methods [23]. The ensemble method provides an optimized prediction by combining the results obtained from the individual learning methods. The main component of our proposed MPF is the combination of KPCA and the accumulator module which is implemented using machine learning methods Multiple Linear Regression (MLR)[24], NN and SVM. The learning algorithm is given below for MPF.

Step 1: Apply different ML methods such as SVM, NN and ANFIS as discussed in sections 3.1, 3.2 , and 3.4 and save the prediction to be used as input.

Step 2: The predictions from the ANFIS, SVM and NN are pre-processed and multicollinearity was removed from them by using KPCA as shown in equation 6

$$
\left[P C 1_{i}, P C 2_{i}, P C 3_{i}\right]=\operatorname{KPCA}\left(N N_{i}, S V M_{i}, A N F I S_{i}\right)
$$

Step 3: The computed principal components obtained from KPCA are used as the independent variables as shown in the equations 7,8 and 9.These independent variables along with the computed predictions are used to fit the MLR and to find the optimal settings for the NN and SVM.

$$
\begin{aligned}
& M P F_{-} v a l u e_{-} M L_{(i)}=\beta_{0}+\beta_{1} P C 1_{i}+\beta_{2} P C 2_{i}+\beta_{3} P C 3_{i}+\text { Error } \\
& M P F_{-} v a l u e_{-} N N_{(i)}=N N_{-} \text {algo }\left(P C 1_{i}, P C 2_{i}, P C 3_{i}\right) \\
& M P F_{-} v a l u e_{-} S V M_{(i)}=S V M_{-} \text {algo }\left(P C 1_{i}, P C 2_{i}, P C 3_{i}\right)
\end{aligned}
$$

Then to compute the performance of the MPF on the test data, independent variables of the test data are also normalized based on therespective mean and standard deviation of training and validation data variables, its kernel matrix is computed and the principal components of the computed kernel matrix are computed using the Eigen-vectors of the training and validation data. 


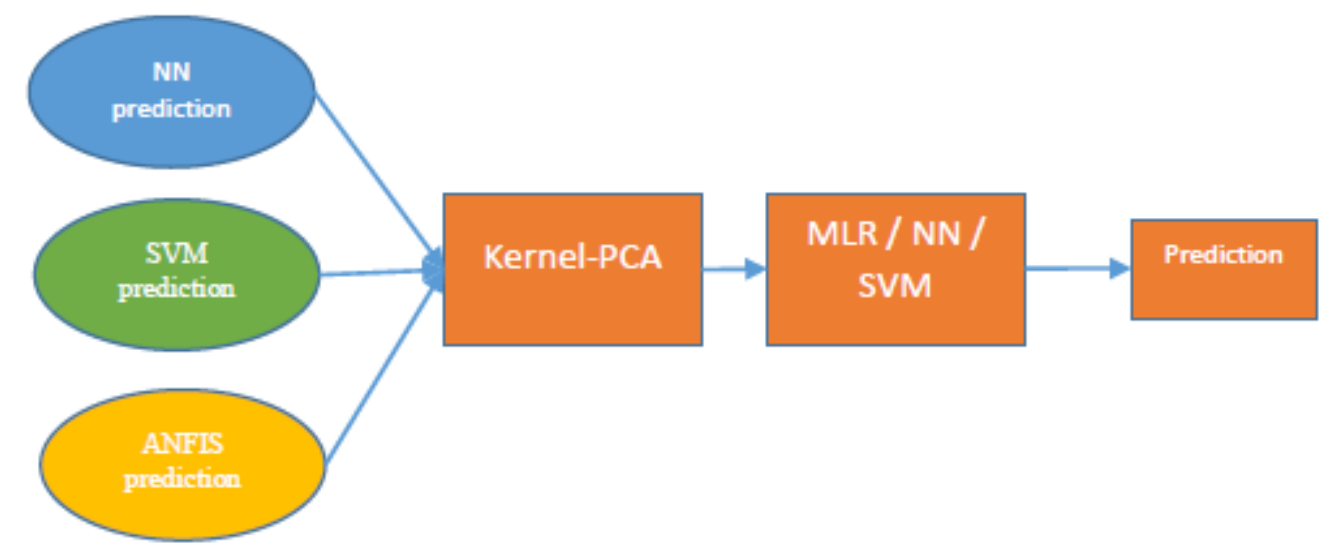

Figure 2 - Proposed Meta Prediction Function (MPF)

To make a comparison with classical machine learning techniques, the test data results of these three MPFs were compared with the best results of the SVM, NN and ANFIS obtained in sections $3.1,3.2$ and 3.4 respectively.

\section{EXPERIMENTS}

In order to evaluate our proposed MPF, we checked its performance on three different datasets related to different fields of actual production data.

The first dataset is related to plastic deformation. It contains 4 variables and 1248 measurements. The dependent variable is the measured flow stress, while the three independent variables are the temperature, the strain and the strain rate during the plastic deformation.

The second dataset is related to the pressure strength of green molding sand. It consists of 5 variables and 1076 measurements. The dependent variable is the green compressive strength, while the 4 independent variables are water content, carbon content, bentonite and clay content.

The third dataset is related to predicting the expected final cost of an ordered product. It consists of 11 variables and 4878 measurements. The dependent variable is the final cost, while the independent variables include delivery weight, material number, length, width, height, molding process, primer, iron ratio and two time related parameters.

To perform the experiments. the input data was divided into three parts. $60 \%$ of the input data was used for training and $20 \%$ was used for validation of the selected machine learning methods to select their respective optimal parameter values and $20 \%$ of the data was used for testing. The results of these methods for test data are shown in section 4.2.

During testing of MPF, the predictions from ANFIS, SVM and NN were transformed using KPCA.

\subsection{Performance Evaluation Measure}

To check the performance of the selected machine learning methods, three different error measures were computed namely Root Mean Square Error (RMSE), Relative Root Mean Square 
Error (RRMSE) and Symmetric Mean Absolute Percentage Error (SMAPE), which are defined as

$$
\begin{aligned}
& R M S E=\sqrt{\frac{1}{n} \sum_{i=1}^{n}\left|O_{i}-P_{i}\right|^{2}} \\
& R R M S E=\sqrt{\frac{1}{n} \sum_{i=1}^{n} \frac{\left|O_{i}-P_{i}\right|^{2}}{\left|O_{i}\right|^{2}}} \\
& \text { SMAPE }=\frac{100 \%}{n} \sum_{i=1}^{n} \frac{\left|O_{i}-P_{i}\right|}{\left(\left|O_{i}\right|+\left|P_{i}\right|\right) / 2}
\end{aligned}
$$

where $n$ is the total number of patterns. $O_{i}$ is the computed output and $P_{i}$ is the predicted output from the used machine learning method.

\subsection{Results and Discussion}

\subsubsection{Results for dataset Flow Stress}

First dataset we used to observe our method performance contains actual measurements from metal forming experiments.

For NN, same settings were used as described in the section 3.2 For SVM, Gaussian kernel with sigma $(\sigma)$ value of 0.5 produced best results.

Since the number of independent variables is smaller in this instance, after computing the principal components, all three of them were selected, covering $100 \%$ of the variance in the data. For ANFIS, Linear kernel along with subtractive clustering (radius: 0.2 ) produced best results as shown in the Table 1 below.

Table 1: Flow stress - Machine learning tool results

\begin{tabular}{|c|c|c|c|}
\hline \multirow{2}{*}{ Error type } & \multicolumn{3}{|c|}{ Learning Methods } \\
\cline { 2 - 4 } & NN & SVM & ANFIS \\
\hline RRMSE & 0.0266 & 0.0336 & 0.0033 \\
\hline SMAPE & 5.0818 & 9.4270 & 0.9259 \\
\hline RMSE & 21.878 & 26.4546 & 2.6550 \\
\hline
\end{tabular}

The following Table 2 shows that the results of the MPF as described in Section 3.5. 
Table 2: Flow stress - MPF results

\begin{tabular}{|l|c|c|c|}
\hline \multirow{2}{*}{ Error Type } & \multicolumn{3}{|c|}{ Kernel: Polynomial } \\
\cline { 2 - 4 } & NN & SVM & MR \\
\hline RRMSE & 0.0033 & 0.0033 & 0.0033 \\
\hline SMAPE & 0.9569 & 0.9225 & 0.9278 \\
\hline RMSE & 2.6397 & 2.6454 & 2.6533 \\
\hline
\end{tabular}

For all the considered error measures, the results as shown in Figure 3 clearly show that the combination of KPCA and ANFIS as well as the MPF with MLR outperformed NN as well as SVM.

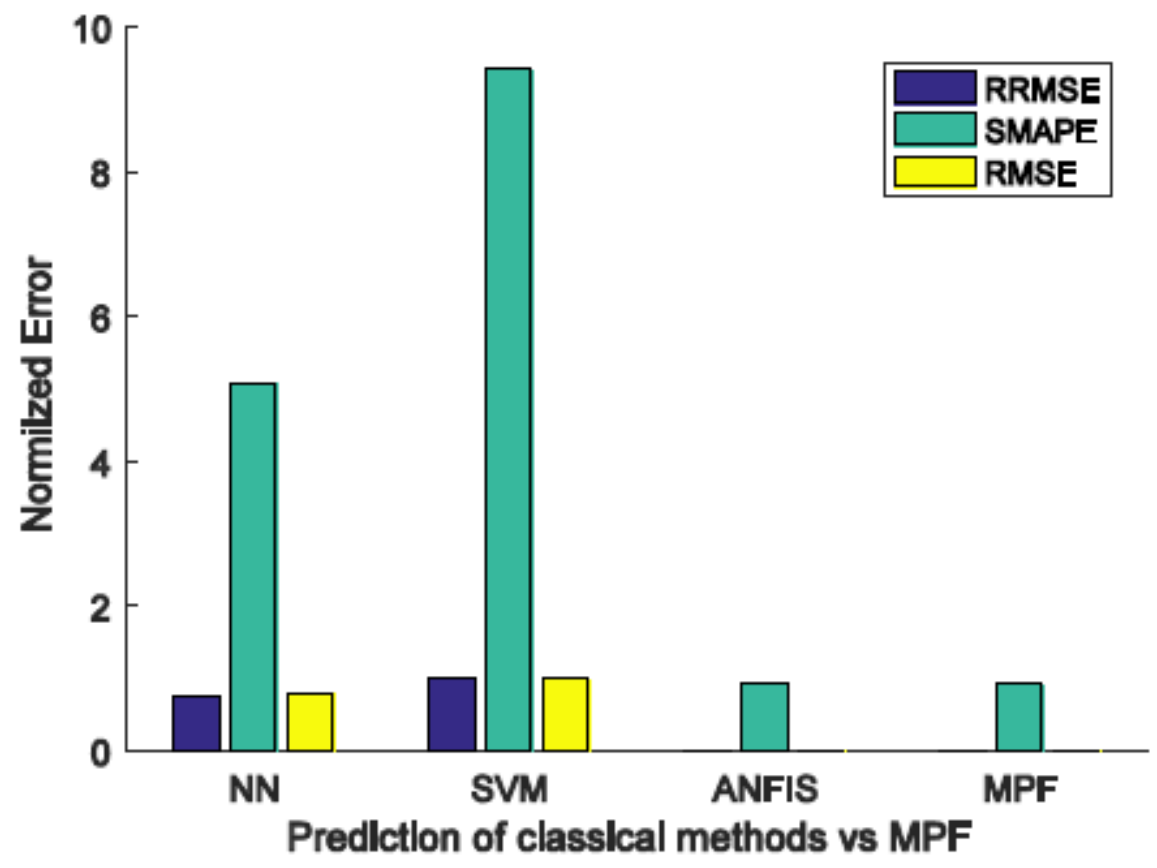

Figure 3: Results of all prediction methods for Flow Stress dataset

\subsubsection{Results for dataset Pressure strength of green molding sand}

Second dataset we used to compare the introduced methodology results is related to computing the pressure strength of green molding sand.

For NN, same settings were used as described in the section 3.2 For SVM, Gaussian kernel with sigma $(\sigma)$ value of 5.0 produced best results.

For this dataset also, all four principal components were selected covering $100 \%$ of the variance. For ANFIS, Linear kernel along with subtractive clustering (radius: 0.3) produced best results. Computed results are shown in Table 3 below. 
Table 3: Pressure shock resistance - Machine learning tools results

\begin{tabular}{|c|c|c|c|}
\hline \multirow{2}{*}{ Error type } & \multicolumn{3}{|c|}{ Learning Methods } \\
\cline { 2 - 4 } & $\mathrm{NN}$ & SVM & ANFIS \\
\hline RRMSE & 0.0425 & 0.0449 & 0.0426 \\
\hline SMAPE & 4.4149 & 4.5869 & 4.3690 \\
\hline RMSE & 1.1688 & 1.2348 & 1.1708 \\
\hline
\end{tabular}

Again, for appropriate parameters, the results of the MPF as shown in the table 4 outperform all of the separate prediction results on the test data.

Table 4: Pressure shock resistance - MPF results

\begin{tabular}{|l|c|c|c|}
\hline \multirow{2}{*}{ Error Type } & \multicolumn{3}{|c|}{ Kernel: Polynomial } \\
\cline { 2 - 4 } & NN & SVM & MR \\
\hline RRMSE & 0.0481 & 0.0434 & 0.0422 \\
\hline SMAPE & 4.9242 & 4.4232 & 4.3410 \\
\hline RMSE & 1.3227 & 1.1944 & 1.1615 \\
\hline
\end{tabular}

As we can see in the Figure 4, our proposed MPF outperforms all the individual learning methods.

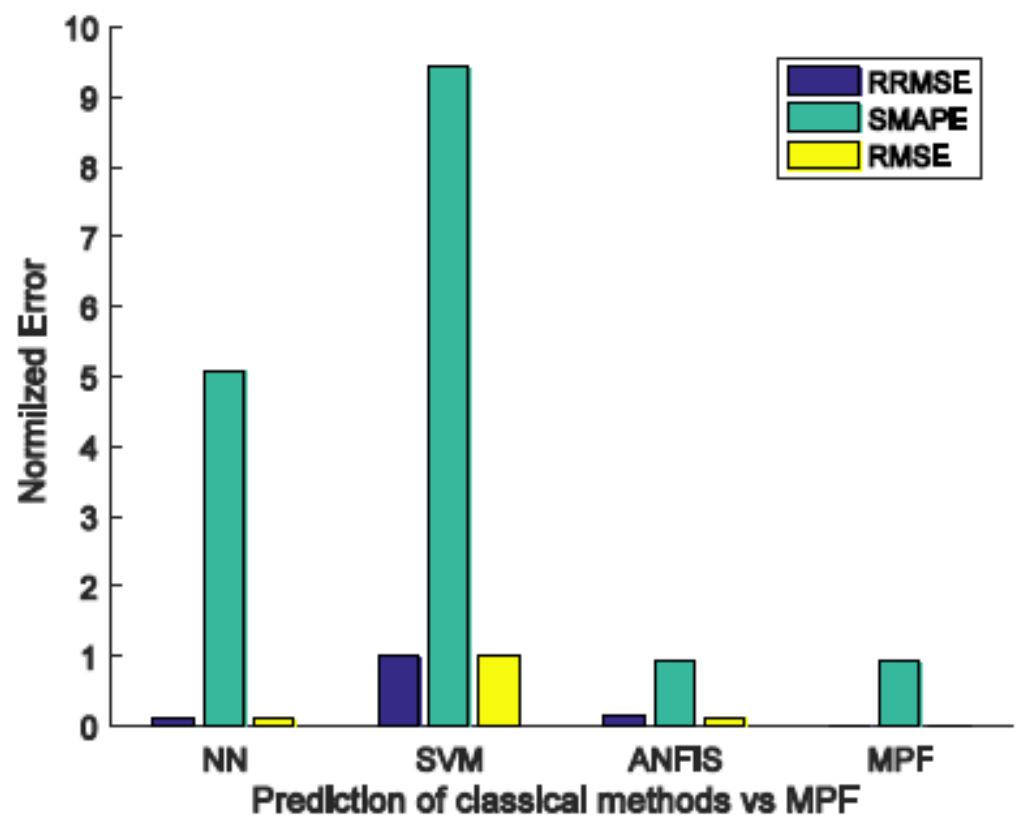

Figure 4: Results of all prediction method for Pressure Strength dataset 


\subsubsection{Results for dataset Final cost}

Final cost dataset contains data recorded from actual production environment and is used to predict the expected final costs of the new ordered product.

For NN, same settings were used as described in the section 3.2 For SVM, Gaussian kernel with sigma $(\sigma)$ value of 5.0 produced best results.

In this case, seven out of ten principal components were selected covering $95.91 \%$ of the variance in the original data. For ANFIS, Linear kernel along with subtractive clustering (radius: 0.2) produced best results. Results are shown in Table 5 below.

Table 5: Final cost - Machine learning tool results

\begin{tabular}{|c|c|c|c|}
\hline \multirow{2}{*}{ Error type } & \multicolumn{3}{|c|}{ Learning Methods } \\
\cline { 2 - 4 } & NN & SVM & ANFIS \\
\hline RRMSE & 0.0143 & 0.0157 & 0.0233 \\
\hline SMAPE & 9.9220 & 21.1273 & 23.9995 \\
\hline RMSE & 347.7719 & 382.5654 & 565.8913 \\
\hline
\end{tabular}

In this case, the prediction provided by the ANFIS in combination with KPCA was not able to achieve the accuracy of the classical methods. However, the MPF performed best in comparison with all the individual methods as shown in the Table 6 below. The ensemble method with MR produced best predictions.

Table 6: Final costs - MPF results

\begin{tabular}{|l|c|c|c|}
\hline \multirow{2}{*}{ Error Type } & \multicolumn{3}{|c|}{ Kernel: Polynomial } \\
\cline { 2 - 4 } & NN & SVM & MR \\
\hline RRMSE & 0.0139 & 0.0218 & 0.0140 \\
\hline SMAPE & 10.9912 & 66.8833 & 8.5959 \\
\hline RMSE & 336.4586 & 529.2121 & 339.2884 \\
\hline
\end{tabular}

Figure 5 given below shows the effectiveness of the MPF in comparison with the performance of SVM and NN. 


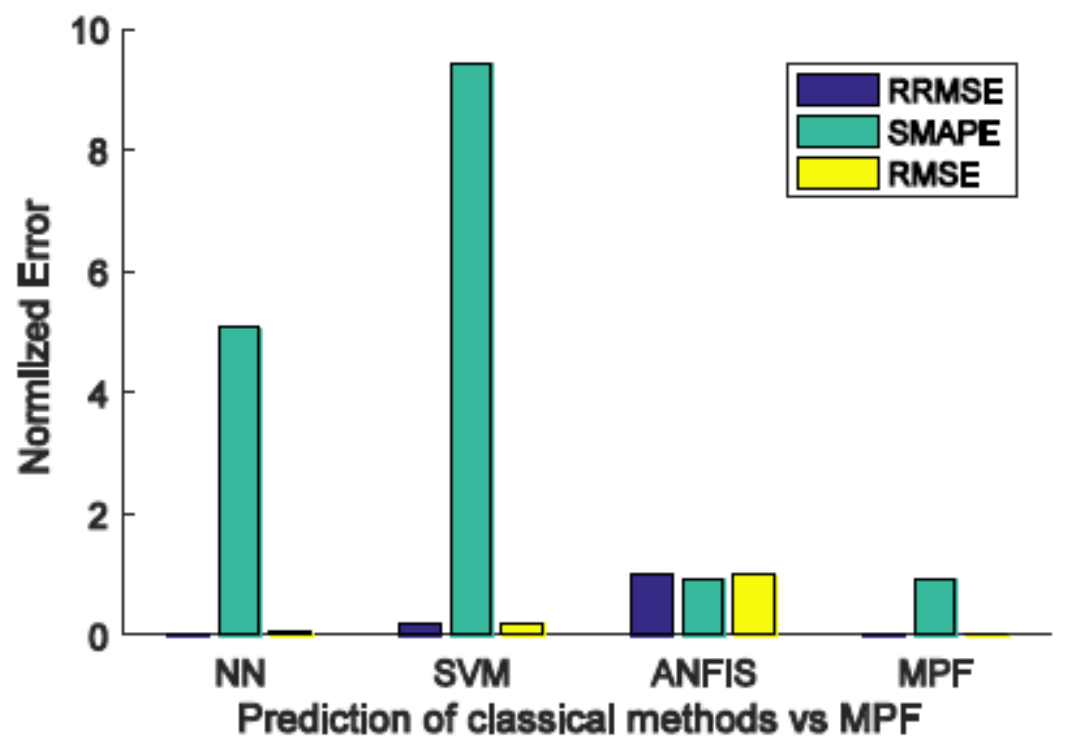

Figure 5: Results of all prediction methods for Final Costs dataset

For the test data, a random sample of prediction results from all the prediction methods used in this work are shown in figure 6. Close observation reveals that the MPF is performing better in comparison with other prediction methods. In Figure 7, which only shows the prediction by MPF, clearly indicates that the MPF is able to predict the test data very accurately.

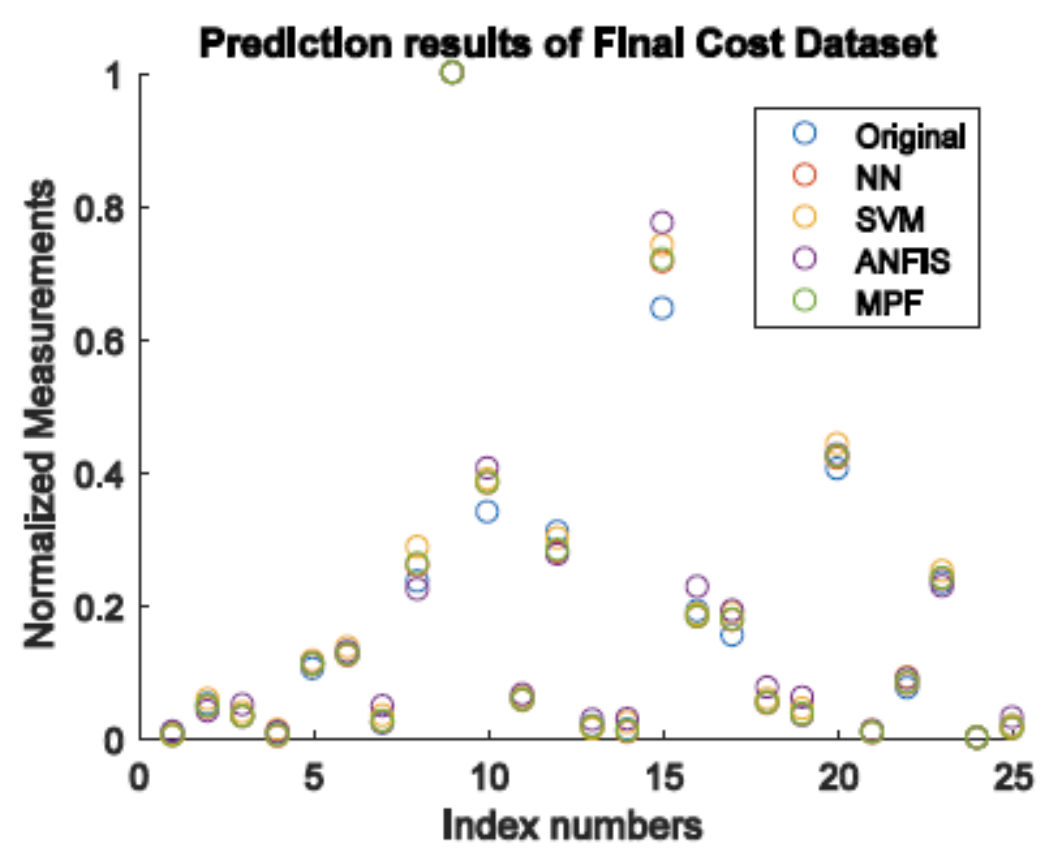

Figure 6: Predictions of sample of measurements for all prediction methods 


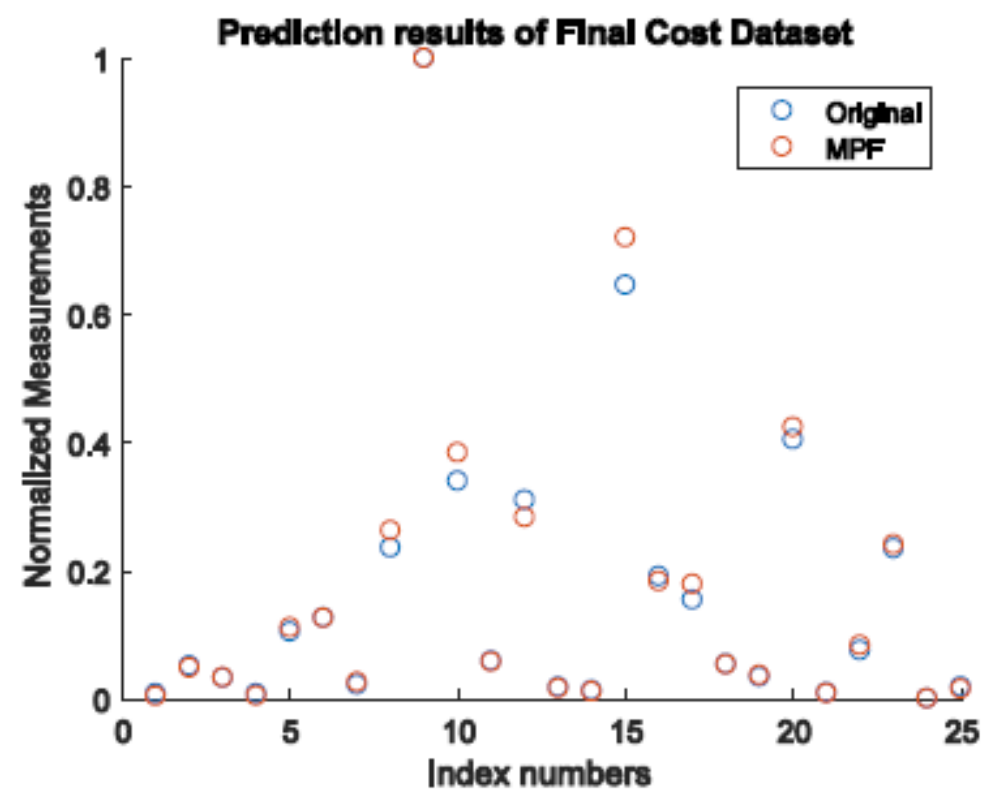

Figure 7: Predictions of sample of measurements for Meta-Prediction Function

\section{CONCLUSION AND FUTURE WORK}

In this paper, we introduced a novel methodology which combines results of different learning methods for quality prediction in production processes. Overall based on RMSE, SMAPE and RRMSE measures, our proposed MPF outperformed the other individual learning methods. Results show that the novel approach of transforming the results of different machine learning methods using KPCA and again applying learning techniques to the obtained data returned very good predictions. Also, it is shown that for the MPF, the combination of a linear kernel for KPCA with MLR produced the best results. For future work, the proposed methodology can be transferred for real time monitoring of production processes to identify parameter deviations which produce faulty parts.

In the future, we will also explore the use of other machine learning methods such as Decision Trees [25] and Bayesian Networks [26] and incorporate them in MPF. Finally, we note that usage of our MPF is not only limited for fault prediction of foundry data, but can also be used for forecasting cost, expenditure or sales prices of production process.

\section{REFERENCES}

[1] Ding, S. X., Yin, S., Peng, K., Hao, H., \& Shen, B. (2013). A novel scheme for key performance indicator prediction and diagnosis with application to an industrial hot strip mill. IEEE Transactions on Industrial Informatics, 9(4), 2239-2247.

[2] Neely, A. (1999). The performance measurement revolution: why now and what next?. International journal of operations \& production management, 19(2), 205-228.

[3] Enkel, E., Perez-Freije, J., \& Gassmann, O. (2005). Minimizing market risks through customer integration in new product development: learning from bad practice. Creativity and Innovation Management, 14(4), 425-437. 
[4] Kan, S. H., Basili, V. R., \& Shapiro, L. N. (1994). Software quality: an overview from the perspective of total quality management. IBM Systems Journal, 33(1), 4-19.

[5] Sosna, Marc, Rosa Nelly Trevinyo-Rodríguez, and S. Ramakrishna Velamuri. "Business model innovation through trial-and-error learning: The Naturhouse case." Long range planning 43.2 (2010): 383-407.

[6] Shingo, S., \& Dillon, A. P. (1989). A study of the Toyota production system: From an Industrial Engineering Viewpoint. CRC Press.

[7] Dilts, D. M., Boyd, N. P., \& Whorms, H. H. (1991). The evolution of control architectures for automated manufacturing systems. Journal of manufacturing systems, 10(1), 79-93.

[8] Kang, S., Kim, E., Shim, J., Cho, S., Chang, W., \& Kim, J. (2017). Mining the relationship between production and customer service data for failure analysis of industrial products. Computers \& Industrial Engineering, 106, 137-146.

[9] Fayyad, U., Piatetsky-Shapiro, G., \& Smyth, P. (1996). The KDD process for extracting useful knowledge from volumes of data. Communications of the ACM, 39(11), 27-34.

[10] Ribeiro, L., \& Barata, J. (2011). Re-thinking diagnosis for future automation systems: An analysis of current diagnostic practices and their applicability in emerging IT based production paradigms. Computers in Industry, 62(7), 639-659.

[11] Schölkopf, Bernhard; and Smola, Alexander J.;" Learning with Kernels”, MIT Press, Cambridge, MA, 2002, ISBN 0-262-19475-9.

[12] Suykens, J. A., \& Vandewalle, J. (1999). Least squares support vector machine classifiers. Neural processing letters, 9(3), 293-300.

[13] Lawrence, Jeanette, "Introduction to Neural Networks", California Scientific Software Press 1994, ISBN 1-883157-00-5.

[14] Craven, M. W., \& Shavlik, J. W. (1997). Using neural networks for data mining. Future generation computer systems, 13(2-3), 211-229.

[15] Jang, J-SR. "ANFIS: Adaptive-Network-based Fuzzy Inference System." IEEE transactions on systems, man, and cybernetics 23.3 (1993): 665-685.

[16] B. Scholkopf, A. Smola, and K. Muller. "Nonlinear component analysis as a kernel eigenvalue problem". Neural Computation, 10(5):1299-1319, 1998.

[17] Yang, J., Frangi, A. F., Yang, J. Y., Zhang, D., \& Jin, Z. (2005). KPCA plus LDA: a complete kernel Fisher discriminant framework for feature extraction and recognition. IEEE Transactions on pattern analysis and machine intelligence, 27(2), 230-244.

[18] Leha, A., Pangercic, D., Rühr, T., \& Beetz, M. (2009, September). "Optimization of simulated production process performance using machine learning". In Emerging Technologies \& Factory Automation, 2009. ETFA 2009. IEEE Conference on (pp. 1-5). IEEE.

[19] Ashour, M. W., Khalid, F., Halin, A. A., \& Abdullah, L. N. (2015, October). "Machining process classification using PCA reduced histogram features and the Support Vector Machine". In Signal and Image Processing Applications (ICSIPA), 2015 IEEE International Conference on (pp. 414-418). IEEE. 
[20] Yuan, Y., Zhang, H. T., Wu, Y., Zhu, T., \& Ding, H. (2016). Bayesian learning-based model predictive vibration control for thin-walled workpiece machining processes. IEEE/ASME Transactions on Mechatronics.

[21] Rostami, H., Blue, J., \& Yugma, C. (2016, December). Equipment Condition Diagnosis and Fault Fingerprint Extraction in Semiconductor Manufacturing. In Machine Learning and Applications (ICMLA), 2016 15th IEEE International Conference on (pp. 534-539). IEEE.

[22] H.R. d. N. Costa and A. La Neve, "A study on the application of regression trees and adaptive neurofuzzy inference system in glass manufacturing process for packaging," 2016 Annual Conference of the North American Fuzzy Information Processing Society (NAFIPS), El Paso, TX, USA, 2016, pp. $1-4$.

[23] Dietterich, Thomas G. "Ensemble methods in machine learning." International workshop on multiple classifier systems. Springer Berlin Heidelberg, 2000.

[24] Aiken, L. S., West, S. G., \& Pitts, S. C. (2003). Multiple linear regression. Handbook of psychology.

[25] Safavian, S. R., \& Landgrebe, D. (1991). A survey of decision tree classifier methodology. IEEE transactions on systems, man, and cybernetics, 21(3), 660-674.

[26] Jansen, R., Yu, H., Greenbaum, D., Kluger, Y., Krogan, N. J., Chung, S., ... \& Gerstein, M. (2003). A Bayesian networks approach for predicting protein-protein interactions from genomic data. science, 302(5644), 449-453.

\section{AUTHORS}

Saad Bashir Alvi is currently a Ph.D. student and a research associate in the Institute for Technologies of Metals at the University of Duisburg-Essen. He has previously worked as a software engineer and received his M.Sc. from University of Bonn, Germany in 2013. His research interests include machine learning and Neural Networks with special emphasis on comparative studies in predictive analytics and focus on dimension reduction as well as neuro fuzzy modeling with applications to production processes.

Robert Martin received his Ph.D. from the University of Duisburg-Essen University, Germany in 2016 and is currently working at the Institute for Technologies of Metals in Duisburg.

Prof. Johannes Gottschling is working at the Institute for Technologies of Metals at the University of Duisburg-Essen and is head of the chair "Mathematics for Engineers".
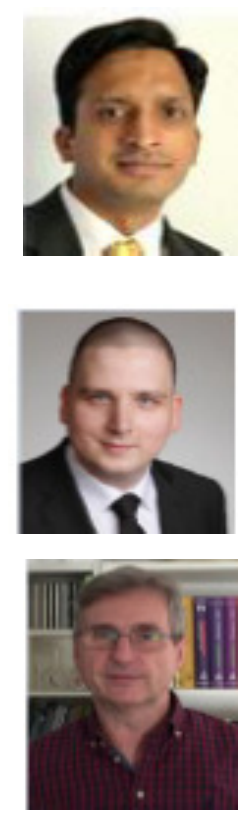